\title{
Clinical scenarios of quadruplet pregnancy by transfer of two blastocysts-A case report
}

\author{
Hossain Amjad, Havemann Dara, Castle Debra, Phelps John \\ Department of Obstetrics and Gynecology, University of Texas Medical Branch, Galveston, USA \\ Email: amhossai@utmb.edu
}

Received 30 January 2012; revised 29 February 2012; accepted 11 March 2012

\begin{abstract}
Blastocyst transfer is advocated to reduce the risk of multiple gestations in pregnancies by assisted reproduction. Nevertheless, there remains the rare inherit possibility of embryo splitting that can result in monozygotic twins leading to high-order multiples. Also, when a patient is found to have a higher gestation than the number of embryos transferred it calls into question the IVF facility's competency and credibility. The case report presented such a rare phenomenon of embryo splitting and the clinical consequences precipitated from it. In the patient, two blastocysts were transferred 5 days after transvaginal oocyte retrieval. The pregnancy confirming hCG test did not predict high-order multiples in this case. Early ultrasonography documented trichorionic-quadramnionic gestation. Selective reduction of the monochorionic twins was done at 11 weeks. Dichorionic twin gestation continued uneventful until 22 weeks at which point the patient experienced preterm premature rupture of membranes with subsequent delivery. The pathology report confirmed trichorionic-quadramnionic gestation. The mode of splitting was different for the two embryos one leading to monozygotic dichorionic and the other to monozygotic monochorionic. Furthermore, the implantation was also apparently asynchronous with one implanted considerable later than the other. The first $\beta$-hCG value seemingly did not represent the entire initial implementation events otherwise the value would be higher carrying the early signal of highorder multiple.
\end{abstract}

Keywords: Blastocyst; Monozygotic Twinning; Quadruplets; IVF

\section{INTRODUCTION}

In this case report, we present a patient who conceived a trichorionic-quadramnionc gestation after undergoing IVF with transfer of 2 day -5 blastocysts. Furthermore, we hypothesize and discuss the possible etiologies lead- ing to this pregnancy. A 25-year-old nulligravida patient presented to our infertility clinic. During the course of her workup, the patient was diagnosed with PCOS with insulin resistance and occlusion of her right fallopian tube, while her partner was diagnosed with oligozoospermia. Treatment was initiated with ovulation induction IUI cycles, and after 3 failed cycles, the patient and her husband chose to proceed with IVF. The patient was started on a long Lupron (Abbot Laboratories, Abbot Park, IL, USA) protocol for ovarian stimulation with Gonal-F (EMD Serono, Rockland, MA, USA) and Menopur (Ferring Pharmaceuticals, Parsippany, NJ, USA) for 10 days followed by triggering with HCG 10,000 U IM. On the day of the retrieval, 13 oocytes were obtained; 11 were mature (MII) and 2 were immature (MI). All 13 oocytes underwent ICSI, of which 10 exhibited fertilization. Embryo transfer of 2 grade 3AA blastocysts was performed with ultrasound guidance on day 5 .

\section{DISCUSSION}

The $\beta$-hCG value recorded for the patient was not indicative of high-order multiples, as the value of $120 \mathrm{mIU} / \mathrm{ml}$ at 13 days post retrieval in our patient fell in the reported range of singleton to twin gestation. The serum first $\beta$-hCG reported to be in the range of $62 \pm 41$ and $109 \pm$ 55 for singleton and twin gestation, respectively $[1,2]$. If all 4 implantations had occurred at the same time, the $\mathrm{hCH}$ value likely would have been higher than that which we measured. Asynchronous embryo implantation thus very likely may have occurred in this case. Zhang et al. showed decreased $\beta$-hCG levels in day 5 blastocyst transfers compared to day 3 embryo transfer [2]. At 39 days post retrieval, transvaginal ultrasound findings showed 3 gestational sacs and four fetal poles, all with positive cardiac activity. More specifically, there were 2 separate sacs immediately adjacent to each other that by ultrasound appeared to have part of their gestational sac in common, sharing a confluent wall. Each of these 2 gestational sacs contained 1 embryo. The third gestational sac was completely separate from the other 2 and contained 2 embryos. The patient was referred to a ma- 
ternal fetal medicine (MFM) specialist who confirmed a trichorionic-quadramnionic pregnancy with cardiac activity of 4 independent fetal poles.

After counseling of the maternal and fetal risks from this type of high-order pregnancy, the patient decided to undergo multi-fetal selective reduction procedure, with a plan for reduction of the monochorionic twins. The reduction was performed by the MFM specialist. The remaining dichorionic-diamnionic twin gestation continued uneventfully until 22 weeks, at which time the patient experienced preterm premature rupture of membranes with subsequent labor and vaginal delivery of 2 nonviable male infants. The final pathology report of the placental membranes showed a trichorionic, quadramniotic quadruplet placenta. All 4 products of conception were males. The patient in this case did have a familial history of twinning. In what follows, we present our case and discuss potential etiologies of trichorionic-quadramniotic gestation after transfer of 2 day- 5 blastocysts.

Our primary explanation is that splitting of both of the transferred blastocysts occurred; however, the time-frame and mode of splitting was likely not the same between the 2 blastocysts. Splitting of the inner cell mass and trophoectoderm of one blastocyst likely occurred, resulting in dichorionic-diamnionic twins. The second blastocyst likely underwent splitting of the inner cell mass only, maintaining a common trohpoectoderm shared by the 2 embryos (splits), thus forming monochorionic-diamnionic twins. Current literature shows that monozygotic multiples occur from embryo splitting; however, such splitting may occur at the preimplantation stage, during implantation, or post implantation [3-5]. The ultrasound at 39 days postretrieval revealed dichorionic-diamnionic twins (2 sacs immediately adjacent to each other with a confluent wall) along with the monochorionic-diamnionic twins in a separate location within the endometrial cavity. Embryo splitting into twins is earlier for dichorionic diamniotic twins as compared to monochorionic diamniotic twins, making an asynchronous embryo implantation possible. This explanation of the events that caused this quadruplet pregnancy is in accordance with the existing classical embryo splitting models proposed in the literature [6-10].

Our second consideration is that the patient may have naturally conceived and become pregnant after the transfer of 2 blastocysts. At the time of the oocyte retrieval, a number of follicles on the left ovary were not aspirated secondary to poor positioning of the ovary and an inability to reach all follicles. The patient and her husband were counseled after the procedure that not all oocytes were retrieved and that they should abstain from intercourse as there was the potential for natural conception. Although the patient denied having had intercourse, if the patient and her husband were to have had intercourse, a resulting natural pregnancy could have occurred following the transfer of the 2 blastocysts, leading to a trichorionic-quadramnionic quadruplet pregnancy [11]. Again, the patient in this case report did have a history of familial twinning, where the propensity for twinning can be inherited through both paternal and maternal lines [12].

Our third consideration as to the cause of this quadruplet pregnancy would be accidental transfer of more embryos than the patient consented for. This hypothesis is highly unlikely, as it is normal laboratory procedure for a 2-person visual confirmation of the number of embryos drawn into the catheter at the time of embryo transfer. Furthermore, no accounting discrepancies were encountered after the embryo transfer occurred. All embryos were present and accounted for after the embryo transfer, minus the 2 blastocysts that were transferred. Nevertheless, any time a pregnancy is of a higher order than the number of embryos transferred, the facility's credibility and competency may be drawn into question.

\section{CONCLUSION}

When a pregnancy results in high-order multiples, there is increased risk of morbidity and mortality to the mother and fetuses. This case report has reviewed different possible etiologies, including splitting of both transferred embryos, simultaneous natural conception, and accounting errors leading to transfer of more than 2 embryos. Although good faith attempts are made to avert highorder multiple pregnancy by limiting the number of embryos transferred to 1 or 2 , as seen in this case report, high-order multiple pregnancies are still possible.

\section{REFERENCES}

[1] Bjercke, S., Tanbo, T., Dale, P., Morkrid, L. and Abyholm, T. (1999) Human chorionic gonadotropin concentrations in early pregnancy after in-vitro fertilization. Human Reproduction, 14, 1642-1646. doi:10.1093/humrep/14.6.1642

[2] Zhang, X., Barnes, R., Confino, E., Milad, M., Puscheck, E. and Kazer, R. (2003) Delay of embryo transfer to day 5 results in decreased initial serum $\beta$-hCG levels. Fertility and Sterility, 80, 1359-1363. doi:10.1016/S0015-0282(03)02201-5

[3] Abusgeika, N., Salha, O., Sharma, V. and Brinsden, P. (2000) Monozygotic twinning and IVF/ICSI treatment: A report of 11 cases and review of literature. Human Reproduction Update, 6, 396-403. doi:10.1093/humupd/6.4.396

[4] Zikopoulos, K. (2004) Quintuplet pregnancy following transfer of two blastocysts: Case report. Human Reproduction, 19, 325-327. doi:10.1093/humrep/deh084

[5] Hall, J.G. (2003) Twinning. Lancet, 362, 735-743. doi:10.1016/S0140-6736(03)14237-7 
[6] Tulchinsky, D., Tulchinsky, A., Falcone, V., Nash, H. and Brown, K. (1996) Delayed embryo implantation following in vitro fertilization and embryo transfer. Journal of Assisted Reproduction and Genetics, 13, 536-539. doi:10.1007/BF02066604

[7] Vela, G., Luna, M., Barritt, J., Sandler, B. and Copperman, A.B. (2011) Monozygotic pregnancies conceived by in vitro fertilization: Understanding their prognosis. Fertility and Sterility, 95, 606-610. doi:10.1016/i.fertnstert.2010.04.001

[8] Silva, S., Martins, Y., Matias, A. and Blickstein, I. (2011) Why are monozygotic twins different? Journal of Perinatal Medicine, 39, 195-202. doi:10.1515/JPM.2010.140

[9] Scott, L. (2002) The origin of monozygotic twinning.
Reproductive Biomedicine Online, 5, 276-284. doi:10.1016/S1472-6483(10)61833-0

[10] Blickstein, I. (2005) Estimation of iatrogenic monozygotic twinning rate following assisted reproduction: Pitfalls and caveats. American Journal of Obstetrics and Gynecology, 192, 365-368. doi:10.1016/j.ajog.2004.11.012

[11] Milki, A.A., Hinckley, M.D., Grumet, F.C. and Chitkara, U. (2001) Concurrent IVF and spontaneous conception resulting in a quadruplet pregnancy. Human Reproduction, 16, 2324-2326. doi:10.1093/humrep/16.11.2324

[12] Parisi, P., Gatti, M., Prinzi, G., Caperna, G. (1983) Familial incidence of twinning. Nature, 304, 626-628. doi: $10.1038 / 304626 \mathrm{a} 0$ 